\title{
Monadic Power Set Theories in Kleisli Categories
}

\author{
Jiří Močkoř \\ Institute for Research and Applications of Fuzzy Modeling \\ University of Ostrava \\ Centre of Excellence IT4Innovations \\ 30. dubna 22, 70103 Ostrava 1, Czech Republic, jiri.mockor@osu.cz
}

\begin{abstract}
In this paper, we follow up on the wellknown interpretation of fuzzy relations as morphisms in Kleisli category defined in the category of sets using a suitable monad. This Kleisli category thus becomes a relational variant of the classical category of fuzzy sets. This interpretation and the associated construction of, e.g., various transformation operators, is often used not only in fuzzy set theory but also in computer science. Using this principle we create in the paper a relational variant of a given Kleisli category which will be defined again using a suitable monad in the original Kleisli category.
\end{abstract}

Keywords: Lattice-valued fuzzy sets, categoris, monad theory, Kleisli categories.

\section{Introduction}

It is well known that fuzzy relations, i.e., fuzzy sets in Cartesian products of two (or more) sets, play in the theory of fuzzy sets an important role. Fuzzy relations occur in a number of constructions in fuzzy set theory, such as fuzzy order relations, fuzzy equivalence relations, approximation of fuzzy sets using fuzzy relations, etc., and at the same time fuzzy relations are an integral part of many applications. It is only a formal statement that fuzzy relations that are defined in a Cartesian product of sets, i.e. $R: X \times Y \rightarrow[0,1]$, can be also defined as a mapping $\bar{R}: X \rightarrow[0,1]^{Y}$, where $\bar{R}(x)(y)=R(x, y)$. In many cases, it is sometimes even more convenient to enter fuzzy relations in this second way as mappings. A typical example might be the theory of soft sets or fuzzy soft sets $[6,7,8,13,17,18]$, which is based entirely on fuzzy relations in this second form, i.e., as mappings. It follows from an interpretation of soft sets or fuzzy soft sets, which are standardly defined as mappings $X \rightarrow 2^{Y}$ or $X \rightarrow[0,1]^{Y}$ (or, generally, $X \rightarrow T(Y)$ ), where $X$ is a set of criteria and $Y$ is a set of objects to be evaluated by these criteria.

Although the expression of a relation or fuzzy relation in the form of a mapping $X \rightarrow T(Y)$ is only a trivial transformation, this representation of relations has become the basis of a very complex theory used not only in fuzzy sets theory but also in other areas such as computer science. This theory is the theory of monads in categories. Monads in a category were introduced by several authors, (for introduction see $[4,9]$ ) and have been used in fuzzifying mathematical objects, including power set structures of fuzzy sets $[10,11,15,16]$ or fuzzy automata $[1,2,3,12]$. The idea of using monads for fuzzyfication is based on extension of objects $X$ of a category $\mathbf{K}$ to another object $T(X) \in \mathbf{K}$. These new objects may be regarded as objects of clouds of fuzzy states. A relation between $X$ and $T(X)$ is described by a morphism $\eta: X \rightarrow T(X)$, representing crisp states in the object of fuzzy states. Then a fuzzy morphism $f: X \rightsquigarrow Y$ is simply a morphism $f: X \rightarrow T(Y)$ (i.e., a special relation) in the category $\mathbf{K}$ and a composition of fuzzy morphisms is defined by a special operation $\diamond$. The result of these constructions is a triple $\mathbf{T}=(T, \diamond, \eta)$, which Manes [9] called a fuzzy theory and which, in fact, is a monad. A system of objects of a category $\mathbf{K}$ with fuzzy morphisms and a composition $\diamond$ of fuzzy morphisms is then a Kleisli category $\mathbf{K}_{\mathbf{T}}$ of a monad $\mathbf{T}$ in the category $\mathbf{K}$ [9]. This category represents a relational variant of the category $\mathbf{K}$, where instead of standard morphisms, relations $X \rightarrow T(Y)$ are used.

In this paper we deal with the following problem. If $\mathbf{K}$ is a category with a monad $\mathbf{T}$, the Kleisli category $\mathbf{K}_{\mathbf{T}}$ is a category with "standard" morphisms $X \rightsquigarrow Y$. How it would be possible to define a "relational" version of this category $\mathbf{K}_{\mathbf{T}}$, where morphisms from $X$ to $Y$ are in a form of relations $X \rightarrow \widetilde{T}(T(Y))$ for some monad $\widetilde{\mathbf{T}}$ in $\mathbf{K}_{\mathbf{T}}$ ? In the fuzzy set theory there are many examples of structures which can be defined as such morphisms. The simplest example of a relation $X \rightarrow[0,1]^{[0,1]^{X}}$ can 
be interpreted as a relationship $R$ between elements $x \in$ $X$ and fuzzy sets $f \in[0,1]^{X}$ defined by $R(x, f)=f(x)$. Another example from the fuzzy soft sets we present in Section 4.

In Section 3 we present a general construction of a relational variant of Kleisli category $\mathbf{K}_{\mathbf{T}}$; more precisely, using a monad $\mathbf{T}$ in a category $\mathbf{K}$ we construct a monad $\widetilde{\mathbf{T}}$ in a Kleisli category $\mathbf{K}_{\mathbf{T}}$ which allows to construct Kleisli category $\left(\mathbf{K}_{\mathbf{T}}\right)_{\widetilde{\mathbf{T}}}$ representing a relational version of $\mathbf{K}_{\mathbf{T}}$. We also investigate relationships between a category $\mathbf{K}$ with a monad $\mathbf{T}$ and a category $\mathbf{K}_{\mathbf{T}}$ with the monad $\widetilde{\mathbf{T}}$

\section{Preliminaries}

In this section we introduce principal notions and categories based on fuzzy sets which we use in the paper. A principal lattice structure used in fuzzy set theory in the paper is a complete residuated lattice (see e.g. [14]), i.e. a structure $\mathscr{L}=\left(L, \wedge, \vee, \otimes, \rightarrow, 0_{L}, 1_{L}\right)$ such that $(L, \wedge, \vee)$ is a complete lattice, $\left(L, \otimes, 1_{L}\right)$ is a commutative monoid with operation $\otimes$ isotone in both arguments and $\rightarrow$ is a binary operation which is residuated with respect to $\otimes$, i.e.

$$
\alpha \otimes \beta \leq \gamma \quad \text { iff } \quad \alpha \leq \beta \rightarrow \gamma
$$

where $\leq$ is the order relation in $L$ defined by the lattice structure of $L$ and $0_{L}$ and $1_{L}$ are the smallest and the greatest elements of $L$, respectively. If $\mathscr{L}$ is a complete residuated lattice, an $\mathscr{L}$-fuzzy set in a crisp set $X$ is a $\operatorname{map} f: X \rightarrow L$.

A set with $\mathscr{L}$-fuzzy similarity relation (or $\mathscr{L}$-set) is a couple $(A, \delta)$, where $A$ is a set and $\delta: A \times A \rightarrow L$ is a map such that

(a) $(\forall x \in A) \quad \delta(x, x)=1$,

(b) $(\forall x, y \in A) \quad \delta(x, y)=\delta(y, x)$,

(c) $(\forall x, y, z \in A) \quad \delta(x, y) \otimes \delta(y, z) \leq \delta(x, z)$ (generalized transitivity)

An $\mathscr{L}$-fuzzy set $s: X \rightarrow L$ is extensional in an $\mathscr{L}$-set $(X, \delta)$, if $s(x) \otimes \delta(x, y) \leq s(y)$ holds for all $x, y \in X$. By $F(X, \delta)$ we denote the set of all extensional $\mathscr{L}$-fuzzy sets in $(X, \delta)$. In the paper we use some standard categories with (sometimes special) maps as morphisms. Namely,

1. Category Set of sets as objects with mappings as morphisms.

2. Category $\operatorname{Set}(\mathscr{L})$ with $\mathscr{L}$-sets $(X, \delta)$ as objects and with $f:(X, \delta) \rightarrow(Y, \gamma)$ as morphisms, where $f: X \rightarrow Y$ is a map and it satisfies $\delta(x, y) \leq$ $\gamma(f(x), f(y)), x, y \in X$.

As we mentioned in the introduction, the main tool from the category theory we will use is a monad in a category. More precisely, we use an extension which is the result of linking the theory of monads $[4,9]$ with the theory of power set objects $[15,16]$. The result of this connection is a construction in a category theory called a monadic power set structure, which is defined as follows:

Definition 2.1 A structure $\mathbf{T}=(T, \diamond, \eta, W)$ is called $a$ monadic power set theory in a category $\mathbf{K}$, if

1. $T: \operatorname{obj}(\mathbf{K}) \rightarrow \operatorname{obj}(\mathbf{K})$ is mapping between objects of $\mathbf{K}$,

2. $W: \mathbf{K} \rightarrow$ Set is a forgetful functor,

3. For arbitrary object $X$ in $\mathbf{K}$, a structure of a complete $\bigvee$-semilattice is defined on a set $W(T(X))$,

4. For K-morphisms $f: X \rightarrow T(Y)$ and $g: Y \rightarrow T(Z)$ there exists their composition $g \oslash f: X \rightarrow T(Z)$, (called the Kleisli composition) which is associative,

5. For arbitrary $\mathbf{K}$-morphisms $f, f^{\prime}: X \rightarrow T(Y)$ and $g, g^{\prime}: Y \rightarrow T(Z)$, the following implications hold

$$
\begin{aligned}
& W(g) \leq_{Y} W\left(g^{\prime}\right) \Rightarrow W(g \diamond f) \leq_{Z} W\left(g^{\prime} \diamond f\right), \\
& W(f) \leq_{Y} W\left(f^{\prime}\right) \Rightarrow W(g \diamond f) \leq_{Z} W\left(g \diamond f^{\prime}\right),
\end{aligned}
$$

where $\leq_{Y}, \leq_{Z}$ are pre-order relations defined point-wise from ordering on $W(T(Y))$ or $W(T(Z))$, respectively.

6. $\eta$ is a system of $\mathbf{K}$-morphisms $\eta_{X}: X \rightarrow T(X)$, for any object $X$ of $\mathbf{K}$,

7. For any $\mathbf{K}$-morphism $f: X \rightarrow Y$, the $\mathbf{K}$-morphism

$$
f_{T}^{\rightarrow}:=\eta_{Y} \cdot f \diamond 1_{T(X)}: T(X) \rightarrow T(Y)
$$

is such that $W\left(f_{\mathbf{T}}^{\rightarrow}\right)$ is also $\bigvee$-preserving map with respect to ordering defined in 3 , where $1_{T(X)}$ is the identity $\mathbf{K}$-morphism $T(X) \rightarrow T(X)$ in $\mathbf{K}$ and "." is a composition of morphisms in a category $\mathbf{K}$.

8. For any K-morphism $f: X \rightarrow T(Y), \eta_{Y} \diamond f=f$ holds,

9. $\diamond$ is compatible with composition of $\mathbf{K}$ morphisms, i.e., for K-morphisms $f: X \rightarrow Y$, $g: Y \rightarrow T(Z)$, we have $g \diamond\left(\eta_{Y} . f\right)=g . f$.

Let us consider the following classical examples of a monadic power set theory. 
Example 2.1 [15] The monad $\mathbf{P}=\left(P, \diamond, \eta, 1_{\text {Set }}\right)$ in the category Set is defined by

1. For each object $X \in \operatorname{Set}, P(X)=2^{X}$,

2. For each object $X \in$ Set, $\eta_{X}: X \rightarrow P(X)$ by $\eta_{X}(x)=\{x\}$.

3. For each $f: X \rightarrow P(Y), g: Y \rightarrow P(Z), g \diamond f: X \rightarrow$ $P(Z)$, by

$$
(g \triangleright f)(x)=\bigcup_{y \in f(x)} g(y)
$$

Example 2.2 [16] The structure $\mathbf{Z}=\left(Z, \boxplus, \chi, 1_{\text {Set }}\right)$ in the category Set is defined by

1. $Z: o b j($ Set $) \rightarrow o b j($ Set $)$ is a mapping defined by $Z(X)=L^{X}$ and $1_{\text {Set }}$ : Set $\rightarrow$ Set is the identity functor. On $L^{X}$ the order relation is defined pointwise,

2. For each $X \in$ Set, $\chi^{X}: X \rightarrow Z(X)$ is the characteristic map of elements from $X$, i.e.,

$x, y \in X, \quad \chi^{X}(x)(y)=\chi_{\{x\}}^{X}(y)= \begin{cases}1_{L}, & x=y, \\ 0_{L}, & \text { otherwise } .\end{cases}$

3. For each $f: X \rightarrow Z(Y)$ and $g: Y \rightarrow Z(V)$ in Set, $g \boxplus f: X \rightarrow Z(V)$ is defined by

$$
(g \boxplus f)(x)(z)=\bigvee_{y \in Y} f(x)(y) \otimes g(y)(z) .
$$

Then $\mathbf{Z}$ is a monadic power set theory.

Example 2.3 [11] The monad $\mathbf{G}=\left(G, \bigotimes, \rho, 1_{\operatorname{Set}(\mathscr{L})}\right)$ in the category $\operatorname{Set}(\mathscr{L})$ is defined by

1. The object function $G: \operatorname{Set}(\mathscr{L}) \rightarrow \operatorname{Set}(\mathscr{L})$ is defined by $G(X, \delta)=\left(F(X, \delta), \sigma_{X, \delta}\right)$, where the similarity relation $\sigma$ is defined by

$$
\sigma_{X, \delta}(s, t)=\bigwedge_{x \in X} s(x) \leftrightarrow t(x),
$$

for all $s, t \in F(X, \delta)$, where $\leftrightarrow$ is the bi-residuum operation in $\mathscr{L}$, such that $a \leftrightarrow b=(a \rightarrow b) \wedge(b \rightarrow$ a),

2. For each $(X, \delta) \in \operatorname{Set}(\mathscr{L}), \rho_{(X, \delta)}:(X, \delta) \rightarrow$ $\mathscr{F}(X, \delta)$ is defined by $\rho_{(X, \delta)}(a)(x)=\delta(a, x)$, for all $a, x \in X$,

3. For each $f:(X, \delta) \rightarrow G(Y, \gamma)$, for each $g$ : $(Y, \gamma) \rightarrow G(Z, \omega)$ in $\operatorname{Set}(\mathscr{L}), g \otimes f:(X, \delta) \rightarrow$ $G(Z, \omega)$ is defined in the same way as in Example 2.2.
For a monadic power set theory $\mathbf{T}$ in a category $\mathbf{K}, \mathbf{K}$ morphisms $X \rightarrow T(Y)$ are called $\mathbf{T}$-relations and are denoted by $X \rightsquigarrow Y$. If $f: X \rightsquigarrow Y$ and $g: Y \rightsquigarrow Z$ are $\mathbf{T}$-relations, their composition is a $\mathbf{T}$-relation defined by $g \diamond f: X \rightsquigarrow Z$. This notion was introduced in the paper of Manes [10] and it was recently proved that it is an universal construction of relations for many fuzzy type structures (e.g., see [11]). Using the notion of a T-relation $X \rightsquigarrow Y$, we can define a notion of a transformation of objects from $T(X)$ to objects from $T(Y)$.

Definition 2.2 [11] Let $R: X \rightsquigarrow Y$ be a T-relation in a category $\mathbf{K}$ with a monadic power set theory $\mathbf{T}=(T, \diamond, \eta, W)$. Then a R-transformation of $o b$ jects from $T(X)$ to objects from $T(Y)$ is a $\mathbf{K}$-morhism $R^{\uparrow}: T(X) \rightarrow T(Y)$, such that $R^{\uparrow}=R \diamond 1_{T(X)}$.

Example 2.4 [11] Recall that an $\mathscr{L}$-valued fuzzy relation from $X$ to $Y$ is an $\mathscr{L}$-valued fuzzy set $R$ in a set $X \times Y$. It is easy to see that $R$ is an $\mathscr{L}$-valued fuzzy relation if and only if $\bar{R}$ is a $\mathbf{Z}$-relation $X \rightsquigarrow Y$, where $\bar{R}(x)(y)=R(x, y)$ and $\mathbf{Z}$ is the monadic power set theory from Example 2.2. In the fuzzy set theory the following transformation operator $R^{\uparrow}: Z(X) \rightarrow Z(Y)$, based on a fuzzy relation $R$ is standardly used:

$$
s \in L^{X}, y \in Y, \quad R^{\uparrow}(s)(y)=\bigvee_{x \in X} s(x) \otimes R(x, y) .
$$

It is easy to see that $R^{\uparrow}=\bar{R} \diamond 1_{Z(X)}=\bar{R}^{\uparrow}$.

Objects of a category $\mathbf{K}$ with $\mathbf{T}$-relations as morphisms define a new category:

Definition 2.3 [5] Let $\mathbf{T}=(T, \diamond, \eta, W)$ be a monadic power set theory in a category $\mathbf{K}$. Kleisli category of a category $\mathbf{K}$ with respect to $\mathbf{T}$ is the category $\mathbf{K}_{\mathbf{T}}$ such that

1. Objects in $\mathbf{K}_{\mathbf{T}}$ are the same as objects of $\mathbf{K}$,

2. Morphisms in $\mathbf{K}_{\mathbf{T}}$ are $\mathbf{T}$-relations $X \rightsquigarrow Y$,

3. Composition of morphisms $f: X \rightsquigarrow Y, g: Y \rightsquigarrow Z$ is $g \diamond f$,

4. For arbitrary object $X \in \mathbf{K}_{\mathbf{T}}$, the unit morphism of $X$ in $\mathbf{K}_{\mathbf{T}}$ is $\eta_{X}: X \rightsquigarrow X$.

Remark 2.1 It should be emphasised that the symbol $f: X \rightsquigarrow Y$ indicates at the same time both a morphism in Kleisli category $\mathbf{K}_{\mathbf{T}}$ and a morphism $X \rightarrow T(Y)$ in a category $\mathbf{K}$. To avoid misunderstandings, if $f: X \rightsquigarrow Y$ is a morphism in $\mathbf{K}_{\mathbf{T}}$, by $f^{*}$ we denote the same morphism $X \rightarrow T(Y)$ in a category $\mathbf{K}$. If $g: Y \rightsquigarrow T(Z)$, then we have $(g \diamond f)^{*}=g^{*} \diamond f^{*}$. 
Moreover, if $\mathbf{T}$ is a monadic power set theory in $\mathbf{K}$, then $T$ can be extended to a functor $T: \mathbf{K} \rightarrow \mathbf{K}$. In fact, if $f: X \rightarrow Y$ is a $\mathbf{K}$-morphism, then $T(f)$ is defined by

$$
T(f)=\eta_{Y} \cdot f \diamond 1_{T(X)}: T(X) \rightarrow T(X) .
$$

\section{Monadic power set theories of Kleisli categories}

Kleisli categories represent a type of a category which is frequently used in computer science and in fuzzy mathematics. Examples of these categories can be found wherever various types of relationships between objects occur and where the composition of these relationships is defined. For this reason, it is interesting and important to decide whether monadic power set theory can also be defined for these Kleisli categories. It subsequently allows not only to define a new concept of a relation in this Kleisli category (i.e., a higher order relation) but also to extend a number of standard constructions, including the approximation of objects using this relation of a new type.

In this Section we prove that for arbitrary Kleisli category $\mathbf{K}_{\mathbf{T}}$, where $\mathbf{T}$ is a monadic power set theory in a category $\mathbf{K}$, there exists a monadic power set theory $\widetilde{\mathbf{T}}$ in Kleisli category $\mathbf{K}_{\mathbf{T}}$. It follows that the Kleisli category $\left(\mathbf{K}_{\mathbf{T}}\right)_{\widetilde{\mathbf{T}}}$ can be considered as a relational variant of the Kleisli category $\mathbf{K}_{\mathbf{T}}$, where instead of morphisms from $\mathbf{K}_{\mathbf{T}}, \widetilde{\mathbf{T}}$-relations as considered.

Theorem 3.1 Let $\mathbf{K}$ be a category and let $\mathbf{T}=$ $(T, \diamond, \eta, W)$ be a monadic power set theory in $\mathbf{K}$. Then there exists a monadic power set theory $\widetilde{\mathbf{T}}=$ $(\widetilde{T}, \square, \xi, V)$ in the Kleisli category $\mathbf{K}_{\mathbf{T}}$.

Proof. The monadic power set theory $\widetilde{\mathbf{T}}$ is defined by

1. For arbitrary object $X$ of $\mathbf{K}_{\mathbf{T}}$, mapping $\widetilde{T}$ : $o b j\left(\mathbf{K}_{\mathbf{T}}\right) \rightarrow \operatorname{obj}\left(\mathbf{K}_{\mathbf{T}}\right)$ is defined by $\widetilde{T}(X)=T(X)$. For this reason instead of $\widetilde{T}(X)$ we simply use $T(X)$.

2. Functor $V: \mathbf{K}_{\mathbf{T}} \rightarrow$ Set is such that $V(X)=W(X)$ and for a morphism $f: X \rightsquigarrow Y$ in $\mathbf{K}_{\mathbf{T}}$ we set $V(f)=W\left(f^{*}\right)$,

3. For arbitrary $\mathbf{K}_{\mathbf{T}}$-morphisms $f: X \rightsquigarrow T(Y)$ and $g: Y \rightsquigarrow T(Z), g \square f: X \rightsquigarrow T(Z)$ is such that

$$
(g \square f)^{*}=g^{*} \diamond 1_{T(Y)} \diamond f^{*}: X \rightsquigarrow T(Z),
$$

4. The $\bigvee$-semilattice structure on $V(T(X))$ is the same as on $W(T(X))$, for arbitrary object $X$,
5. For arbitrary object $X$ in $\mathbf{K}_{\mathbf{T}}, \xi_{X}: X \rightsquigarrow T(X)$ is defined such that $\xi_{X}^{*}=\eta_{T(X)}^{*} \cdot \eta_{X}^{*}: X \rightarrow T(X) \rightarrow$ $T(T(X))$, where "." is the composition of $\mathbf{K}$ morphisms.

We show that this definition of $\widetilde{\mathbf{T}}$ is correct. In fact, for a $\mathbf{K}_{\mathbf{T}}$-morphism $f: X \rightsquigarrow T(Y)$ we have

$$
\begin{gathered}
\left(\xi_{Y} \square f\right)^{*}=\xi_{Y}^{*} \diamond 1_{T(Y)} \diamond f^{*}=\eta_{T(Y)} \cdot \eta_{Y} \diamond 1_{T(Y)} \diamond f^{*}= \\
\eta_{T(Y)} \cdot\left(\eta_{Y} \diamond 1_{T(Y)}\right) \diamond f^{*}=\eta_{T(Y)} \cdot 1_{T(Y)} \diamond f^{*}= \\
\eta_{T(Y)} \diamond f^{*}=f^{*} .
\end{gathered}
$$

Therefore, $f=\xi_{Y} \square f$ and the condition (8) from Definition 2.1 holds. Now, because the composition $\diamond$ is associative, the composition operation $\square$ is also associative, as for arbitrary $\mathbf{K}_{\mathbf{T}}$-morphisms $f: X \rightsquigarrow T(Y)$, $g: Y \rightsquigarrow T(Z), h: Z \rightsquigarrow T(Q)$ follows from the identity

$(h \square(g \square f))^{*}=h^{*} \diamond 1_{T(Z)} \diamond g^{*} \diamond 1_{T(Y)} \diamond f^{*}=((h \square g) \square f)^{*}$.

Hence the condition (4) from Definition 2.1 holds. Now, let $f: X \rightsquigarrow Y$ and $g: Y \rightsquigarrow T(Z)$ be $\mathbf{K}_{\mathbf{T}^{-}}$ morphisms. Then we have

$$
\begin{gathered}
\left(g \square\left(\xi_{Y} \diamond f\right)\right)^{*}=g^{*} \diamond 1_{T(Y)} \diamond\left(\xi_{Y} \diamond f\right)^{*}= \\
g^{*} \diamond 1_{T(Y)} \diamond\left(\eta_{T(Y)} \cdot \eta_{Y} \diamond f^{*}\right)= \\
g^{*} \diamond 1_{T(Y)} \diamond \eta_{T(Y)} \cdot\left(\eta_{Y} \diamond f^{*}\right)= \\
g^{*} \diamond 1_{T(Y)} \diamond \eta_{T(Y)} \cdot f^{*}=g^{*} \diamond\left(1_{T(Y)} \diamond \eta_{T(Y)}\right) \cdot f^{*}= \\
g^{*} \diamond f^{*}=(g \diamond f)^{*} .
\end{gathered}
$$

Therefore, we obtain $g \square\left(\xi_{Y} \diamond f\right)=g \diamond f$ and the condition (9) from Definition 2.1 holds.

Finally, recall that the unit $\mathbf{K}_{\mathbf{T}}$-morphism of an element $T(X)$ is $\eta_{T(X)}$. Hence, according to (7) from Definition 2.1 , for a $\mathbf{K}_{\mathbf{T}}$-morphism $f: X \rightsquigarrow Y$ we have

$$
\begin{gathered}
\left(f_{\widetilde{\mathbf{T}}}^{\rightarrow}\right)^{*}=\left(\left(\xi_{Y} \diamond f\right) \square \eta_{T(X)}\right)^{*}= \\
\left(\xi_{Y} \diamond f\right)^{*} \diamond 1_{T(X)} \diamond \eta_{T(X)}^{*}= \\
\xi_{Y}^{*} \diamond f^{*} \diamond 1_{T(X)} \diamond \eta_{T(X)}^{*}=\eta_{T(Y)} \cdot \eta_{Y} \diamond f^{*} \diamond 1_{T(X)} \diamond \eta_{T(X)}^{*}= \\
\eta_{T(Y)}^{*} \cdot\left(\eta_{Y}^{*} \diamond f^{*}\right) \diamond 1_{T(X)} \diamond \eta_{T(X)}^{*}= \\
\eta_{T(Y)}^{*} f^{*} \diamond 1_{T(X)} \diamond \eta_{T(X)}^{*}= \\
\eta_{T(Y)}^{*} \cdot f^{*} \diamond 1_{T(X)} .
\end{gathered}
$$

Therefore, we have

$$
V\left(f_{\widetilde{\mathbf{T}}}^{\rightarrow}\right)=W\left(\left(f_{\widetilde{\mathbf{T}}}\right)^{*}\right): T(X) \rightarrow T T(Y)
$$

and if we apply the condition (7) from Definition 2.1 to the K-morphism $f^{*}: X \rightarrow T T(Y)$, we obtain that $V\left(f_{\widetilde{\mathbf{T}}}\right)$ is also $\bigvee$-preserving map. Therefore, $\widetilde{\mathbf{T}}$ is a monadic power set theory in Kleisli category $\mathbf{K}_{\mathbf{T}}$. 
Let's try to explain how morphisms look like in the Kleisli category $\left(\mathbf{K}_{\mathbf{T}}\right)_{\widetilde{\mathbf{T}}}$. For this purpose, we use the following symbols for morphisms from $\mathbf{K}, \mathbf{K}_{\mathbf{T}}$ and $\left(\mathbf{K}_{T}\right)_{\widetilde{\mathbf{T}}}$, respectively:

$$
X \rightarrow Y, \quad X \rightsquigarrow Y, \quad X \Rightarrow Y .
$$

As it was mentioned, a morphism $X \rightsquigarrow Y$ in $\mathbf{K}_{\mathbf{T}}$ represents also the morphism $X \rightarrow T(Y)$ in $\mathbf{K}$. Analogously, a morphism $X \Rightarrow Y$ in $\left(\mathbf{K}_{\mathbf{T}}\right)_{\widetilde{\mathbf{T}}}$ represents also the morphism $X \rightsquigarrow \widetilde{T}(Y)=X \rightsquigarrow T(Y)$ in $\mathbf{K}_{\mathbf{T}}$ and this morphism represents also a morphism $X \rightarrow T(T(Y))$ in $\mathbf{K}$. Therefore, by morphisms $X \Rightarrow Y$ in $\left(\mathbf{K}_{T}\right)_{\widetilde{\mathbf{T}}}$ we can consider morphisms $X \rightarrow T(T(Y))$ in $\mathbf{K}$. Using notation from Remark 2.1 , if $R: X \Rightarrow Y$ is a $\left(\mathbf{K}_{\mathbf{T}}\right)_{\widetilde{\mathbf{T}}^{-}}$ morphism, then $R^{*}: X \rightsquigarrow T(Y)$ is a corresponding $\mathbf{K}_{\mathbf{T}^{-}}$ morphism and $R^{* *}: X \rightarrow T(T(Y))$ is a corresponding K-morphism.

The method presented in Theorem 3.1 allows us to create an infinite sequence of categories with higher order relations as morphisms from one category $\mathbf{K}$ with monadic power set theory $\mathbf{T}$, i.e., a sequence of Kleisli categories defined over Kleisli categories. In fact, we can use the following sequence of Kleisli categories

$$
\mathbf{K} \stackrel{\mathbf{T}}{\rightarrow} \mathbf{K}_{\mathbf{T}} \stackrel{\widetilde{\mathbf{T}}}{\rightarrow}\left(\mathbf{K}_{\mathbf{T}}\right)_{\widetilde{\mathbf{T}}} \stackrel{\widetilde{\mathbf{T}}^{\prime}}{\rightarrow}\left(\left(\mathbf{K}_{\mathbf{T}}\right)_{\widetilde{\mathbf{T}}}\right)_{\widetilde{\mathbf{T}}^{\prime}} \rightarrow \text { etc. }
$$

Relationships between pairs of categories from this sequence can be described by morphisms between objects $(\mathbf{K}, \mathbf{T})$ consisting of a category $\mathbf{K}$ and a monadic power set theory $\mathbf{T}$ in this category. This notion was introduced in [12].

Definition 3.1 [12] By a monadic couple we understand the couple $(\mathbf{K}, \mathbf{T})$, where $\mathbf{T}=(T, \diamond, \eta, W)$ is a monadic power set theory in a category $\mathbf{K}$. Let $(\mathbf{L}, \mathbf{R})$ be another monadic couple such that $\mathbf{R}=$ $(R, \square, \mu, V)$. Then $(H, \Phi):(\mathbf{K}, \mathbf{T}) \rightarrow(\mathbf{L}, \mathbf{R})$ is a morphism of monadic couples if

\section{1. $H: \mathbf{K} \rightarrow \mathbf{L}$ is a functor,}

2. $V H(X)=W(X)$ for an arbitrary object $X$ in $\mathbf{K}$,

3. $\Phi: H T \rightarrow R H$ is a natural transformation of a composition of functors,

4. For each morphisms $f: X \rightarrow T(Y), g: Y \rightarrow T(Z)$ in $\mathbf{K}$, the following equality holds:

$$
\Phi_{Z} \cdot H(g \diamond f)=\left(\Phi_{Z} \cdot H(g)\right) \square\left(\Phi_{Y} \cdot H(f)\right),
$$

where . is a composition of $\mathbf{L}$-morphisms in $\mathbf{L}$.
5. For arbitrary object $X$ in $\mathbf{K}$, the following diagram of $\mathbf{L}-m o r p h i s m s$ commutes:

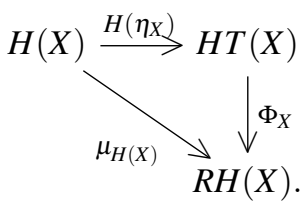

In the following proposition we show the basic relationship between monadic pairs $(\mathbf{K}, \mathbf{T})$ and $\left(\mathbf{K}_{\mathbf{T}}, \widetilde{\mathbf{T}}\right)$.

Proposition 3.1 There exists a morphism

$$
(H, \Phi):(\mathbf{K}, \mathbf{T}) \rightarrow\left(\mathbf{K}_{\mathbf{T}}, \widetilde{\mathbf{T}}\right)
$$

of monadic pairs from Theorem 3.1.

Proof. The morphism $(H, \Phi)$ is defined by

1. $H: \mathbf{K} \rightarrow \mathbf{K}_{\mathbf{T}}$ is defined by

(a) $H(X)=X$ for objects from $\mathbf{K}$,

(b) For a K-morphism $f: X \rightarrow Y$, a $\mathbf{K}_{\mathbf{T}^{-}}$ morphism $H(f): X \rightsquigarrow Y$ is such that $H(f)^{*}=\eta_{Y} \cdot f: X \rightarrow T(Y)$, where "." is the composition of morphisms in $\mathbf{K}$.

2. For an object $X$ in $\mathbf{K}, \Phi_{X}: H T(X) \rightsquigarrow \widetilde{T} H(X)$ is a $\mathbf{K}_{\mathbf{T}}$-morphism $T(X) \rightsquigarrow T(X)$ such that $\Phi_{X}^{*}=$ $\eta_{T(X)}: T(X) \rightarrow T(T(X))$.

We prove that $H$ is a functor. Let $f: X \rightarrow Y$ and $g$ : $Y \rightarrow Z$ be $\mathbf{K}$-morphisms. Since the composition of $\mathbf{K}_{\mathbf{T}^{-}}$ morphisms is $\diamond$, to prove that $H$ is a functor we need to show that $H(g . f)=H(g) \diamond H(f)$. In fact, we have

$$
\begin{aligned}
& H(g . f)^{*}=\eta_{Z} \cdot g \cdot f, \\
& (H(g) \diamond H(f))^{*}=\eta_{Z} \cdot g \diamond \eta_{Y} \cdot f=\eta_{Z} \cdot\left(g \diamond \eta_{Y} \cdot f\right)= \\
& \eta_{Z} \cdot\left(g \diamond \eta_{Y}\right) \cdot f=\eta_{Z} \cdot g \cdot f, \\
& H\left(1_{X}\right)=\eta_{X}=1_{H(X)} \text {. }
\end{aligned}
$$

Further, let $f: X \rightarrow Y$ be a $\mathbf{K}$-morphism. To prove that $\Phi$ is a natural transformation, we need to prove that the following diagram of $\mathbf{K}_{\mathbf{T}}$-morphisms commutes:

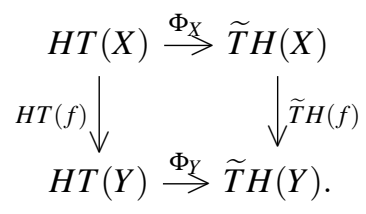

According to Remark 2.1 we have

$$
\begin{gathered}
\left(\Phi_{Y} \diamond H T(f)\right)^{*}=\eta_{T(Y)} \diamond H(T(f))^{*}= \\
\eta_{T(Y)} \diamond \eta_{T(Y)} \cdot\left(\eta_{Y} \cdot f \diamond 1_{T(X)}\right)=\eta_{T(Y)} \cdot\left(\eta_{Y} \cdot f \diamond 1_{T(X)}\right) .
\end{gathered}
$$


On the other hand, we have

$$
\begin{aligned}
& \left(\widetilde{T} H(f) \diamond \Phi_{X}\right)^{*}=\widetilde{T}\left(\eta_{Y} \cdot f\right)^{*} \diamond \eta_{T(X)}= \\
& \left(\left(\xi_{Y} \diamond \eta_{Y \cdot f)} \square \eta_{T(X)}\right)^{*} \diamond \eta_{T(X)}=\right. \\
& \xi_{Y} \cdot f \diamond 1_{T(X)} \diamond \eta_{T(Y)}=\eta_{T(Y)} \cdot \eta_{Y} \cdot f \diamond 1_{T(X)} \diamond \eta_{T(X)}= \\
& \eta_{T(Y)} \cdot\left(\eta_{Y \cdot f} \diamond 1_{T(X)}\right) \diamond \eta_{T(X)}=\eta_{T(Y)} \cdot\left(\eta_{Y \cdot f} \diamond 1_{T(X)}\right) .
\end{aligned}
$$

Therefore, the diagram commutes and $\Phi$ is a natural transformation. Further, we prove that the equality (2) holds. Let $f: X \rightarrow T(Y)$ and $g: Y \rightarrow T(Z)$ be Kmorphisms. We have

$$
\begin{gathered}
\left(\Phi_{Z} \diamond H(g \diamond f)\right)^{*}=\eta_{T(Z)} \diamond H(g \diamond f)^{*}=H(g \diamond f)^{*}= \\
\eta_{T(Z)} \cdot(g \diamond f)=\eta_{T(Z)} \cdot g \triangleright f .
\end{gathered}
$$

On the other hand, we have

$$
\begin{gathered}
\left(\left(\Phi_{Z} \diamond H(g)\right) \square\left(\Phi_{Y} \diamond H(f)\right)\right)^{*}=(H(g) \square H(f))^{*}= \\
\left(\left(\eta_{T(Z)} \cdot g\right) \square\left(\eta_{T(Y)} \cdot f\right)\right)^{*}=\eta_{T(Z)} \cdot g \diamond 1_{T(Y)} \diamond \eta_{T(Y)} \cdot f= \\
\eta_{T(Z)} \cdot g \diamond\left(1_{Y(Y)} \diamond \eta_{T(Y)}\right) \cdot f=\eta_{T(Z)} \cdot g \diamond f .
\end{gathered}
$$

Therefore, the equality (2) holds. Finally, we show that the diagram from condition 5 in Definition 3.1, commutes. In fact, the commutativity of this diagram is transformed to the equality $\Phi_{X} \diamond H\left(\eta_{X}\right)=\xi_{H(X)}=$ $\xi_{X}$ and this equality follows directly from equalities $\Phi_{X} \diamond H\left(\eta_{X}\right)=\eta_{T(X)} \diamond \eta_{T(X)} \cdot \eta_{X}=\eta_{T(X)} \cdot \eta_{X}=\xi_{X}$. Therefore, $(H, \Phi)$ is a morphism of monadic pairs.

From Proposition 3.1 and Theorem 3.1 it follows that we can construct a sequence of morphisms between monadic pairs

$$
\left.(\mathbf{K}, \mathbf{T}) \stackrel{(H, \Phi)}{\longrightarrow}\left(\mathbf{K}_{\mathbf{T}}, \widetilde{\mathbf{T}}\right) \stackrel{\left(H^{\prime}, \Phi^{\prime}\right)}{\longrightarrow}\left(\left(\mathbf{K}_{\mathbf{T}}\right)_{\widetilde{\mathbf{T}}}, \widetilde{\mathbf{T}}^{\prime}\right)\right) \rightarrow \text { etc. }
$$

\section{Examples}

In the following example we show how the category $\left((\text { Set })_{\mathbf{Z}}\right)_{\widetilde{\mathbf{z}}}$ can be described in a simpler and more illustrative way.

Example 4.1 Let us consider the following category K.

1. Objects of $\mathbf{K}$ are the same as in the category $\mathbf{S e t}$.

2. K-morphisms $R: X \rightarrow Y$ are $\mathscr{L}$-fuzzy relations from $X$ to $Z(Y)$, i.e., $R: X \times Z(Y) \rightarrow L$.

3. Compositions of $\mathbf{K}$-morphisms $R: X \rightarrow Y$ and $S$ : $Y \rightarrow Q$ is an $\mathscr{L}$-fuzzy relation $S \circ R: X \times Z(Q) \rightarrow$ $L$, defined by

$$
\begin{gathered}
x \in X, \quad t \in Z(Q), \\
S \circ R(x, t)=\bigvee_{s \in Z(Y)} \bigvee_{y \in Y} R(x, s) \otimes s(y) \otimes S(y, t) .
\end{gathered}
$$

4. For an object $X$ in $\mathbf{K}$, the unit $\mathbf{K}$-morphism $1_{X}$ : $X \rightarrow X$ is an $\mathscr{L}$-fuzzy relation $1_{X}: X \times Z(X) \rightarrow L$, such that

$$
x \in X, s \in Z(X), 1_{X}(x, s)= \begin{cases}1_{L}, & \text { if } s=\chi_{\{x\}}, \\ 0_{L}, & \text { otherwise }\end{cases}
$$

It is easy to prove that the category $\mathbf{K}$ is isomorphic to Kleisli category $\left(\operatorname{Set}_{\mathbf{Z}}\right)_{\widetilde{\mathbf{Z}}}$.

Example 4.2 For illustration, we show how morphisms $R:(X, \delta) \Rightarrow(Y, \gamma)$ look in the category $\left(\operatorname{Set}(\mathscr{L})_{\mathbf{G}}\right)_{\widetilde{\mathbf{G}}}$, where $\mathbf{G}$ is a monadic power set theory from Example 2.3. According to Remark 2.1, $R$ is equivalent to the $\mathbf{S e t}(\mathscr{L})$-morphism

$$
\begin{gathered}
\left(R^{*}\right)^{*}:(X, \delta) \rightarrow G(G(Y, \gamma)), \text { where } \\
G(G(Y, \gamma))=G\left(F(Y, \gamma), \sigma_{Y, \gamma}\right)= \\
\left(\left(F\left(F(Y, \gamma), \sigma_{Y, \gamma}\right), \sigma_{F(Y, \gamma), \sigma_{Y, \gamma}}\right) .\right.
\end{gathered}
$$

It follows that the following statements are equivalent.

1. $R:(X, \delta) \Rightarrow(Y, \gamma)$ is a morphism in $\left(\operatorname{Set}(\mathscr{L})_{\mathbf{G}}\right)_{\widetilde{\mathbf{G}}}$,

2. $R: X \times F(Y, \gamma) \rightarrow L$ is an $\mathscr{L}$-fuzzy relation, such that

(a) For arbitrary $x, x^{\prime} \in X$, it holds

$$
\delta\left(x, x^{\prime}\right) \leq \bigwedge_{t \in F(Y, \gamma)} R(x, t) \leftrightarrow R\left(x^{\prime}, t\right)
$$

(b) For arbitrary $x \in X, s, t \in F(Y, \gamma)$, it holds

$$
R(x, s) \otimes \bigwedge_{y \in Y} s(y) \leftrightarrow t(y) \leq R(x, t)
$$

In the next example we show that morphisms $X \Rightarrow Y$ in Kleisli category $\left.(\mathbf{S e t})_{\mathbf{z}}\right)_{\widetilde{\mathbf{Z}}}$ can, in fact, occur even in quite commonly used systems, such as e.g. $\mathscr{L}$-fuzzy soft sets.

Example 4.3 We use a notation from Example 2.2. Recall that an $\mathscr{L}$-fuzzy soft set in a soft universe $(X, K)$ is a pair $(E, s)$, where $X$ is a set of objects, $K$ is a set of criteria, $E \subseteq K$ and $s: E \rightarrow Z(X)$ is a mapping (see, e.g., [6]). It is clear that $\mathscr{L}$-fuzzy soft sets can be equivalently represented as a pair $(E, \bar{s})$, where $\bar{s}: X \rightarrow Z(E)$ is a mapping such that $\bar{s}(x)(e)=s(e)(x)$. In this case, $\bar{s}(x)$ is a fuzzy set in $E$ expressing how the object $x$ satisfies individual criteria from $E$. Unlike the individual criteria $e \in E$, we can also consider criteria, which are represented by fuzzy sets in E. It is natural to call these fuzzy sets as fuzzy criteria for selecting variants from $X$.

Now, if a fuzzy criterion $h \in Z(E)$ is defined, we must determine how a given object $x$ corresponds to $h$. For a 
given $\mathscr{L}$-fuzzy soft set $(E, s)$, object $x \in X$ and a fuzzy criterion $h \in Z(E)$, we need to associate a value in $L$. Equivalently, it means that we need to define a mapping $X \rightarrow Z(Z(E))$. According to Theorem 3.1 and Remark 2.1, this mapping is equivalent to the morphism

$$
R_{(E, s)}: X \Rightarrow E
$$

in the Kleisli category $\left(\operatorname{Set}_{\mathbf{Z}}\right)_{\widetilde{\mathbf{Z}}}$ or, equivalently, to the Setz-morphism $R_{(E, s)}^{*}: X \rightsquigarrow Z(E)$, or, equivalently, the Set-morphism $\left(R_{(E, s)}^{*}\right)^{*}: X \rightarrow Z(Z(E))$ in Set. For example, for $x \in X, h \in Z(E)$ we can set

$$
\left(R_{(E, s)}^{*}\right)^{*}(x)(h)=\bigvee_{e \in E} h(e) \otimes s(e)(x) .
$$

In that case, $\left(R_{(E, s)}^{*}\right)^{*}(x)(h)$ is the value with which the object $x$ corresponds to the fuzzy criterion $h$, provided that the object $x$ is primarily evaluated using a fuzzy soft set $(E, s)$.

In addition, just as we introduced the notion of a fuzzy criterion, we can introduce (at least for some tasks) the notion of a fuzzy portfolio of objects from $X$ as a fuzzy set $g \in L^{X}$, where the value $g(x) \in L$ corresponds to how strongly we consider including object $x$ in our individual selection of suitable objects. It is then possible to solve the problem of how a fuzzy portfolio $g$ of objects corresponds to a fuzzy scenario $h$. For this purpose we can use the morphism $R_{(E, s)}: X \Rightarrow E$ in the Kleisli category $\left(\operatorname{Set}_{\mathbf{z}}\right)_{\widetilde{\mathbf{Z}}}$, which is also a $\mathbf{Z}$ relation $R_{(E, s)}^{*}: X \rightsquigarrow Z(E)$. According to Definition 2.2 , using this $\mathbf{Z}$-relation $R_{(E, s)}^{*}$ we can construct the $R_{(E, s)}^{*}$-transformation of objects from $Z(X)$ to objects $Z(Z(E))$ defined by

$$
\begin{gathered}
R_{(E, s)}^{* \uparrow}: Z(X) \rightarrow Z(Z(E)), \\
g \in Z(X), h \in Z(E), \quad R_{(E, s)}^{*}(g)(h)= \\
R_{(E, s)}^{*} \boxplus 1_{Z(X)}(g)(h)=\bigvee_{x \in X} \bigvee_{e \in E} g(x) \otimes h(e) \otimes s(e)(x) .
\end{gathered}
$$

Hence, $R_{(E, s)}^{*}{ }^{\uparrow}(g)(h)$ represents the value from $L$ describing how fuzzy portfolio g corresponds to the fuzzy scenario $h$.

Example 4.4 The simplest example of a morphism in Kleisli category $\left(\mathbf{S e t}_{\mathbf{Z}}\right)_{\widetilde{\mathbf{Z}}}$ is a calculation of membership functions of $\mathscr{L}$-fuzzy sets. In fact, let $R: X \Rightarrow X$ be a $\left(\text { Set }_{\mathbf{Z}}\right)_{\widetilde{\mathbf{Z}}}$-morphism. Using the notation (1) and Remarks 2.1, this morphism is equivalent to the Setzmorphism $R^{*}: X \rightsquigarrow Z(X)$ and Set-morphism $\left(R^{*}\right)^{*}$ : $X \rightarrow Z(Z(X))$. Now, the mapping $\left(R^{*}\right)^{*}$ can be defined by

$$
x \in X, s \in Z(X), \quad\left(R^{*}\right)^{*}(x)(s)=s(x) \in L .
$$

Therefore, the $\left(\mathbf{S e t}_{\mathbf{z}}\right)_{\widetilde{\mathbf{z}}}$-morphism $R: X \Rightarrow X$ can be identified with the process of calculations of membership functions of $\mathscr{L}$-fuzzy sets from $Z(X)$.

\section{Conclusions}

In this paper, we continued to build relational variants of categories $\mathbf{K}$, i.e., such modifications of a category $\mathbf{K}$, where instead of $\mathbf{K}$-morphisms $f: X \rightarrow Y$ of this category, special relations $R: X \rightsquigarrow Y$ defined by a monad $T$ in this category are considered. Then Kleisli category $\mathbf{K}_{\mathbf{T}}$ of a category $\mathbf{K}$ defined by a monad $\mathbf{T}$ can be considered as a relational variant of a category $\mathbf{K}$. We first presented this type of transformation of a category $\mathbf{K}$ to relational version in [11], where we showed that many standard fuzzy type relations in various categories are in fact relations defined by monads and, therefore, categories with such defined relations as morphisms are isomorphic to Kleisli category.

Although often not explicitly stated, the Kleisli categories themselves are also often used in the fuzzy set theory and their modifications. It is therefore interesting to examine what the relational variants of Kleisli categories look like, i.e., how it is possible to construct Kleisli categories of Kleisli categories using appropriate monads. In the paper we show how to define a monad $\widetilde{\mathbf{T}}$ in a Kleisli category $\mathbf{K}_{\mathbf{T}}$ of a category $\mathbf{K}$ and a monad $\mathbf{T}$, such that $\left(\mathbf{K}_{\mathbf{T}}\right)_{\widetilde{\mathbf{T}}}$ represents a relational variant of the category $\mathbf{K}_{\mathbf{T}}$. We show also some examples of morphisms in this category $\left(\mathbf{K}_{\mathbf{T}}\right)_{\widetilde{\mathbf{T}}}$ and relationship between categories $\mathbf{K}_{\mathbf{T}}$ and $\left(\mathbf{K}_{\mathbf{T}}\right)_{\widetilde{\mathbf{T}}}$.

\section{Acknowledgement}

This work was partly supported from ERDF/ESF project CZ.02.1.01/0.0/0.0/17-049/0008414.

\section{References}

[1] M. Arbib, E. Manes, Fuzzy Morphisms in Automata Theory, Lect. Notes Comp. Sci. 25 (1975), 80-86.

[2] M. Arbib, E. Manes, Machines in a Category: An Expository Introduction, SIAM Rev. 16 (1974), 163-192.

[3] M. Arbib, E. Manes, Fuzzy Machines in a Category, Bull. Austra. Math. Soc. 13 (1975).

[4] H. Herrlich, G.E. Strecker, Category Theory (3rd ed.), Heldermann Verlag Berlin, 2007.

[5] S. Mac Lane, Categories for the Working Mathematician, Graduate Texts in Mathematics 5 (2nd ed.), Springer-Verlag, New York (1998).

[6] P.K. Maji .et al., Fuzzy soft-sets, Journal of Fuzzy Math. 9(3)(2001), 589-602.

[7] P.K. Maji, R. Biswas, A.R. Roy, Soft set theory, Comput. Math. Appl. 45(2003), 555-562. 
[8] P.K. Maji et al., An application of soft sets in a decision making problem, Comput. Math. Appl. 44(2002), 1077-083.

[9] E.G. Manes, Algebraic Theories, Springer Verlag, Berlin, 1976.

[10] E.G. Manes, Book review Fuzzy sets and systems, Theory and applications, Bulletin (New Series) of the American Mathematica Society 7(3)(1982).

[11] J. Močkoř, Fuzzy type relations and transformation operators defined by monads, International Journal of Computational Intelligence Systems 13(1)(2020), 1530-1538.

[12] J. Močkoř, Monads and a common framework for fuzzy type automata, International Journal of General Systems 48(4)(2019), 406-442.

[13] D. Molodtsov, Soft set theory-First results, Comput. Math. Appl. 37(1999), 19-31.

[14] V. Novák, I. Perfilijeva, J. Močkoř, Mathematical principles of fuzzy logic, Kluwer Academic Publishers, Boston, Dordrecht, London (1991).

[15] S.E. Rodabaugh, Relationship of Algebraic Theories to Power Set Theories and Fuzzy Topological Theories for Lattice-Valued Mathematics, International Journal of Mathermatics and Mathematical Sciences 2007, 1-71.

[16] S.E. Rodabaugh, Power set operator foundation for poslat fuzzy set theories and topologies, Höhle,U.,Rodabaugh, S.E. (Eds.), Mathematics of Fuzzy Sets: Logic, Topology and Measure Theory, The Handbook of Fuzzy Sets Series, Vol. 3 , Kluwer Academic Publishers, Boston, Dordrecht (1999), 91-116.

[17] T. Som, On the theory of soft sets, soft relations and fuzzy soft relations, Proceedings of the National Conference on Uncertainty: A Mathematical Approach, Burdwan (India) (2006), 1-9.

[18] Y. Zhang, X. Yuan, Z. Xia, On Fuzzy Soft Relation. In: Cao BY., Wang PZ., Liu ZL., Zhong YB. (eds) International Conference on Oriental Thinking and Fuzzy Logic, Advances in Intelligent Systems and Computing, vol 443(2016), Springer, Cham. 